\title{
Development of a Direct Assessment for Measuring Students' Ability to Make Connections
}

\section{Meg West, Ohio State University}

Meg West is an engineering education graduate student at The Ohio State University. She is a graduate research associate for the Department of Engineering Education.

\section{Dr. Meagan Eleanor Ita, Ohio State University}

Meagan is a Postdoctoral Scholar in the Engineering Education Department at The Ohio State University. She graduated with her Bachelor's (2013) and Master's (2014) in Biomedical Engineering from The Ohio State University and completed her Ph.D. in Bioengineering at the University of Pennsylvania in 2020. Meagan conducted research on the biomechanics and physiology of chronic pain for her doctorate degree and has experience teaching undergraduate first-year engineering and mid-level biomechanics courses. Meagan is currently working with the KEEN partnership at OSU, integrating her interests in STEM education, entrepreneurial partnerships, and community engagement. Meagan values authenticity, connection with others, \& integrity and prioritizes these values as an educator, bioengineer, and scientist.

\section{Laine Rumreich, Ohio State University}

Laine Rumreich is a graduate student studying Computer Science and Engineering at The Ohio State University. She completed her undergraduate research thesis in the Department of Engineering Education and is now a graduate research associate in the department. Her primary research interests are in the areas of coding education and engineering entrepreneurship.

\section{Dr. Rachel Louis Kajfez, Ohio State University}

Dr. Rachel Louis Kajfez is an Assistant Professor in the Department of Engineering Education at The Ohio State University. She earned her B.S. and M.S. degrees in Civil Engineering from Ohio State and earned her Ph.D. in Engineering Education from Virginia Tech. Her research interests focus on the intersection between motivation and identity of undergraduate and graduate students, first-year engineering programs, mixed methods research, and innovative approaches to teaching. She is the faculty lead for the Research on Identity and Motivation in Engineering (RIME) Collaborative.

\section{Dr. Krista M. Kecskemety, Ohio State University}

Krista Kecskemety is an Assistant Professor of Practice in the Department of Engineering Education at The Ohio State University. Krista received her B.S. in Aerospace Engineering at The Ohio State University in 2006 and received her M.S. from Ohio State in 2007. In 2012, Krista completed her Ph.D. in Aerospace Engineering at Ohio State. Her engineering education research interests include investigating first-year engineering student experiences, faculty experiences, and the connection between the two. 


\title{
Developing a Direct Assessment for Measuring Students' Ability to Make Connections
}

\begin{abstract}
The integration of entrepreneurial minded learning (EML) into engineering courses to develop students' entrepreneurial mindset (EM) is growing in popularity through efforts such as the Kern Entrepreneurial Engineering Network (KEEN). As the integration of EML occurs, it is important to assess students' development of an EM to inform the efficacy of course changes. Following KEEN's definition, we operationalize EM as the ability to create value, make connections, and be curious. While we acknowledge the importance of all aspects of EML, in this full paper, we focus on assessing students' ability to make connections. Concept maps have been previously used to assess student development of an EM as a whole, and we believe they can also be used to specifically assess the ability of students to make connections. Specifically, we collected responses from a pre-existing individual concept map activity used in two sections of a first-year engineering course and two sections of an aerospace engineering course at The Ohio State University. A total of 238 responses were collected, 106 responses from the first-year engineering course and 132 responses from the aerospace engineering course. The concept maps were evaluated using the traditional concept map scoring method. Through our analysis, we found no strong correlation between course grades and scores on concept maps developed by students for the course. This result supports moving forward with the concept map scoring methodology without the need for a correction factor related to grades. That said, other results indicated the need for modifications to the concept map instructions and scoring method that accounted for intra-hierarchy connections. In future studies, we will explore these findings further including the possibility of creating a new concept map scoring method with a stronger focus on measuring connections.
\end{abstract}

\section{Introduction}

Many engineering programs are introducing entrepreneurial concepts based on evolving employer expectations as well as student and instructor interests [1]. The benefits of introducing entrepreneurial concepts into engineering coursework are becoming increasingly well known. For example, engineers equipped with an entrepreneurial mindset (EM) have been shown to understand the "bigger picture", recognize opportunities, evaluate markets, and learn from mistakes to create value for themselves, for their employers, and for society [2]. Additionally, entrepreneurial concepts are becoming more highly valued by students, faculty, and administrators. For example, a 2015 survey of ASEE members representing 100 institutions indicated that faculty and administrators strongly agree that engineering students should have access to innovation and entrepreneurship education [3].

The Kern Entrepreneurial Engineering Network (KEEN) is a leader in defining and distributing concepts and course content related to EM in engineering. KEEN is a collaborative network of academic institutions and professors with the shared mission of cultivating the core principles of the EM in their students [2]. This organization guides the network's activity related to curricular development, faculty workshops, and student engagement and have defined the $3 \mathrm{C}$ 's of EM to 
unify their model and educational materials. These 3 C's are curiosity, connections, and creating value.

Due to a nationwide effort to increase evidence of student learning in higher education [4], universities are being pushed to not only integrate EM into their courses but also to measure the impact and effectiveness of the change. Various assessments have been used to evaluate student development of an EM. Brunhaver, Bekki, Carberry, London, and McKenna [5] developed an indirect assessment called the Engineering Student Entrepreneurial Mindset Assessment (ESEMA) that contains 34 items across seven constructs to measure undergraduate engineering students' EM. Their instrument, while designed to measure curiosity, connections, and creating value, contained seven emergent factors identified as Interest, Empathy, Open-Mindedness, Ideation, Altruism, and Help Seeking with the final factor staying unnamed (as the remaining items included in this factor did not converge around an interpretable construct) [5]. Additionally, Li, Harichandran, Carnasciali, Erdil, and Nocito-Gobel [6] created an indirect assessment instrument with 27 items across 10 constructs to measure engineering student EM. Their instrument contained factors more closely related to the 3C's and the engineering skillset put forth by KEEN (i.e. Problem Solving/Logical Thinking, Value Creation, Engaging Stakeholders, Analyze Market Conditions). While such instruments measure EM holistically, Zappe [7] argues that such assessments could lead to construct underrepresentation due to oversimplifying of the sub-constructs that make up EM. As such, she calls for an EM assessment approach that is attribute-focused rather than globally focused [7]. Using KEEN's framework of EM, we have begun developing a set of six assessments to measure engineering students' 3C's individually. At this project's conclusion, each of these three core areas will be measured using one indirect assessment and one direct assessment. This paper focuses on one of those three core areas where a current assessment tool does not exist: measuring students' ability to make connections using concept maps as a direct assessment. Future work and publications will focus on other assessments for the $3 \mathrm{Cs}$ as well as on other aspects of EM.

\section{Background}

At The Ohio State University, we are in the process of incorporating EM into engineering courses and are interested in evaluating whether existing course components and added EM content are aiding student development of an EM, specifically, student ability to make connections. In this context, making connections is defined as the ability to use a systemsthinking approach to connect disparate information from diverse sources, such as engineering courses, non-technical undergraduate courses, media sources, or personal experiences. In the context of a single undergraduate engineering course, making connections involves the students' ability to identify course elements and the connections between explicitly linked content and seemingly disparate curricular components. However, solely measuring a concept as abstract as the ability to make connections can be challenging. We believe concept maps to be one possible method to measure this ability.

Concept maps are visual representations of a person's networked knowledge about a topic and are widely accepted as a tool for formative assessment [8]. The depth and breadth of understanding of a topic is directly related to the complexity of a concept map, and the 
understanding of the subject matter and relationships between sub-topics are represented by the organization of the map. In this way, concept maps can be used to evaluate students' knowledge and understanding $[9,10]$. Concept maps also allow students to illustrate the connections they form between new information and material that has been previously studied, which allows them to think about the underlying structure of what is learned [11].

Concept maps have been used in classrooms as an instructional and assessment tool in a variety of STEM domains, including biology, medicine, and chemistry [12-14]. Concept maps have also served as a tool to evaluate high-level skills such as critical thinking [15] and conceptual math knowledge [16]. Recently, concept maps have been specifically utilized in the realm of EM. For example, Bodnar et al. [17] used concept maps to assess student development of an EM holistically and aimed to capture all three "C"s" in their assessment. The process used by Bodnar et al. [17] was to develop a single master concept map on EM based on individual concept maps developed by 26 EM experts. This master EM concept map was then published as a tool for instructors for use in a formative assessment.

The combined previous usage of concept maps as an EM assessment and the call to assess subconstructs of EM prompted our development of a direct assessment of students' ability to make connections using concept maps. The assessment prompts ask students to create a concept map that relates all of the concepts from their current course to each other.

For this work, we will answer the research question: How can concept maps be used to assess students' ability to make connections? In answering this research question, we explore the benefits and limitations of using a known concept map scoring method for the purpose of widespread use by instructors and teaching assistants with varying levels of content knowledge. We also explore the potential impact of course grades on our ability to assess students' ability to make connections.

\section{Methods}

To explore concept maps as a direct assessment of student ability to make connections, we collected responses from a pre-existing concept map activity used in two sections of a first-year engineering course and two sections of an aerospace engineering course typically taken during the third year at The Ohio State University. These courses were chosen because they were taught by the same instructor and represent two different points of time in an undergraduate curriculum. These courses did not have EM content intentionally included in the course, and thus, any connections students made between topics were without targeted EM interventions. The activity in both courses involved students individually creating a concept map of their course at the end of the semester. A total of 238 responses to the activity were collected consisting of 106 responses from the first-year engineering course and 132 responses from the aerospace engineering course. There was the possibility that student performance in a course may influence their ability to create a concept map about that same course's content. As such, we collected students' final course grades from both courses to determine if a relationship exists between students' ability to make meaningful connections via concept maps and their success in the 
course. All data were collected and analyzed under approved Institutional Review Board procedures.

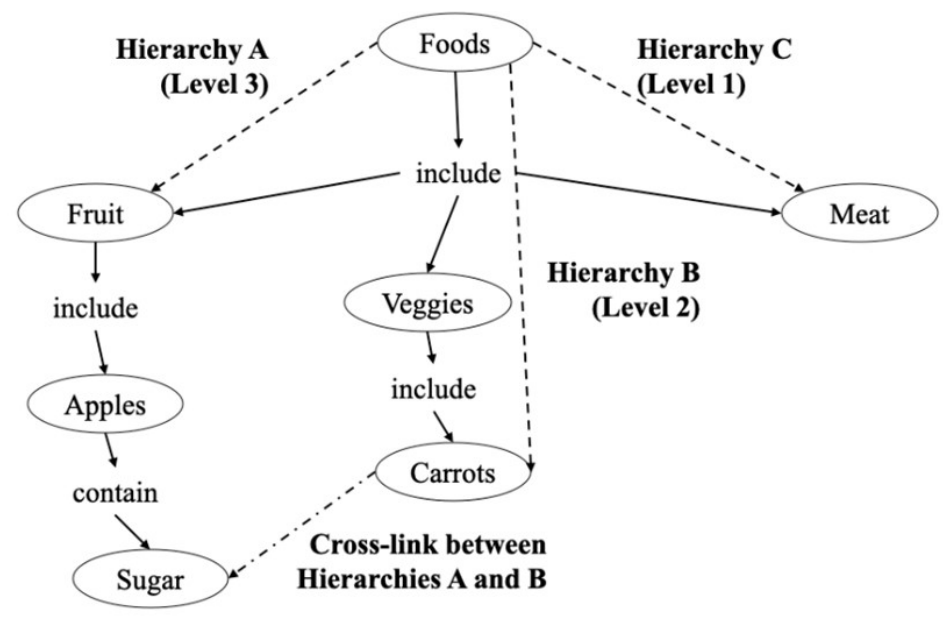

Figure 1. Concept map hierarchies and cross-links inspired by Novak \& Gowin [8] and Watson et al. [18].

The concept maps were evaluated using the traditional concept map scoring, a quantitative method developed by Novak and Gowin [8] and described by Watson, Pelkey, Noyes, and Rodgers [18] for use in engineering education settings. Another concept map scoring method, the holistic method, was considered for use in this study. The holistic concept map scoring method uses a three-point rubric scale to rate the organization, correctness, and comprehensiveness of concept maps [18]. Its qualitative nature allows for subjective rating that could cause variability in scores given across multiple raters. Additionally, it requires the rater to have a firm grasp on the content knowledge being mapped. The traditional concept map scoring method was chosen because of its quantitative nature, it does not require raters to be fluent in the content mapped, and its sub-score that directly measures connections made within a concept map. The traditional scoring method has been found to have acceptable interrater reliability and convergent and divergent validity [18]. The traditional scoring method uses three values, Number of Concepts (NC), Highest Hierarchy (HH), and Number of Cross-links (NCL), to calculate a total concept map score. The NC represents the knowledge breadth sub-score where concepts are the items contained within a boundary/box (excluding the central topic or starting node) [18]. Figure 1 contains a sample concept map that illustrates the three values used in traditional concept map scoring. In the example, the NC is six because "Foods" is the central topic and not counted in the NC. The HH represents the knowledge depth sub-score where a hierarchy is defined by propositions that include the concept map topic (concepts stemming from the central topic) [18]. $\mathrm{HH}$ is the number of concepts in the longest path down a hierarchy. In the example in Figure 1, the $\mathrm{HH}$ is three because Hierarchy A has three concepts which is more that the number of concepts within Hierarchy B or Hierarchy C. The NCL represents the knowledge connectedness sub-score where cross-links are links between concepts in different hierarchies [18]. In Figure 1, the NCL is one (the cross-link between Hierarchy A and Hierarchy B). The total concept map score is the sum of the NC, five times the HH, and ten times the NCL. The total concept map score for the example is 31 . 
We began the scoring process of the collected concept map data with two raters comparing their scores for a subset of the data to ensure interrater reliability. The two raters independently scored ten concept maps. Nine of the ten scores were identical between the two raters. The tenth score differed in the NC score between the two raters due to miscounting the number of the nodes. Throughout the remaining scoring, both raters documented their observations and discussed observation notes about the concept map submissions and the overall scoring process.

One limitation of the traditional scoring method is that it does not include a scoring component for correctness or accuracy of the concept map. In other words, the traditional scoring method does not take into account whether connections are logical or "make sense". For instance, students may make connections between items that are trivial or even unrelated, but these connections are treated no differently from sophisticated connections with the traditional scoring method. Furthermore, since our concept map assignment prompted students to create concept maps specifically on course content, it is possible that concept map scores not only reflect students' ability to make systems-thinking connections in a broad sense, but that scores also reflect student understanding of course content. So, it is possible that concept map scores may be confounded by student understanding of course content.

Given the possibility that student performance in a course may influence their ability to create a concept map about that same course's content, we collected students' final course grades to determine if a relationship exists between students' success in the course (measured by their final course grades) and their ability to make meaningful connections via concept maps (measured by the concept map score); statistical analyses were performed on the data to test this relationship. All statistical analyses were performed with $\alpha=0.05$ using JMP-Prov14 (SAS Institute Inc). Normality was tested using a Shapiro-Wilk goodness-of-fit test for a normal continuous fit on the residuals of the concept map scores and the final course grades. Since a Shapiro-Wilk test revealed non-normal distributions for both concept map scores and final course grades, both metrics were treated as non-normal continuous numeric variables. A non-parametric Spearman's rank correlation test was performed to test the significance of relationships between concept map score and final course grade for each of the first-year and aerospace data, separately. The Spearman's rank coefficient, $\rho$, ranges from -1 (strong negative correlation) to +1 (strong positive correlation), with 0 indicating no relationship.

\section{Results}

We observed several sources of variability across the structures and methodologies that students utilized to develop their concept maps that presented difficulties in using the traditional scoring method. For example, although most concept maps were clear and easy to read, some had no clear central topic. This made it difficult to use the traditional scoring method because all three values (NC, HH, and NCL) needed to calculate the total score are dependent upon how the concepts are connected to the central topic. In the instance where there was no clear central topic, the rater chose one node as the assumed central topic in order to complete the traditional scoring method. Several students also submitted multi-page and hand-written concept maps that were difficult to read and often contained unclear connecting lines. The lines used to connect concepts were often crossing in a manner that made them hard to decipher and required raters to make 
assumptions about the HH and NCL values. Additionally, many concept maps had intrahierarchy connections (connections between concepts in the same hierarchy) that are not counted using the traditional concept map scoring method.

Overall, the average final course grade was lower for the aerospace course than the first-year course (Table 1, Figure 2A). In contrast, the average concept map scores were higher for the aerospace course than the first-year course (Table 1, Figure 2A). Concept map scores for both of

Table 1. Summary data for final course grade and concept map score shown separately for each course dataset.

\begin{tabular}{rc|c|c|c} 
& \multicolumn{2}{c|}{ final course grade } & \multicolumn{2}{c}{ concept map score } \\
\cline { 2 - 5 } & first-year & aerospace & first-year & aerospace \\
\cline { 2 - 5 } minimum & 75.63 & 58.16 & 8 & 31 \\
maximum & 101.50 & 97.77 & 180 & 228 \\
mean & 93.95 & 85.45 & 57 & 97 \\
standard deviation & 5.48 & 7.32 & 30 & 37 \\
\hline \hline
\end{tabular}

the first-year and aerospace data were found to be non-normally distributed $(p<0.001)$ (Figure 2B). In the first-year course, there was a significant $(\mathrm{p}=0.047)$ yet weak positive correlation $(\rho=0.194)$ between final course grade and concept map score (Figure 3$)$. No correlation was found between final course grade and concept map score in the aerospace course $(p=0.105$; $\rho=0.142$ ) (Figure 3). Data sets from both courses contained several outliers as determined by an analysis on the residuals (Figure 3 ), with $n=6$ and $n=7$ outliers observed in the first-year and aerospace courses, respectively.
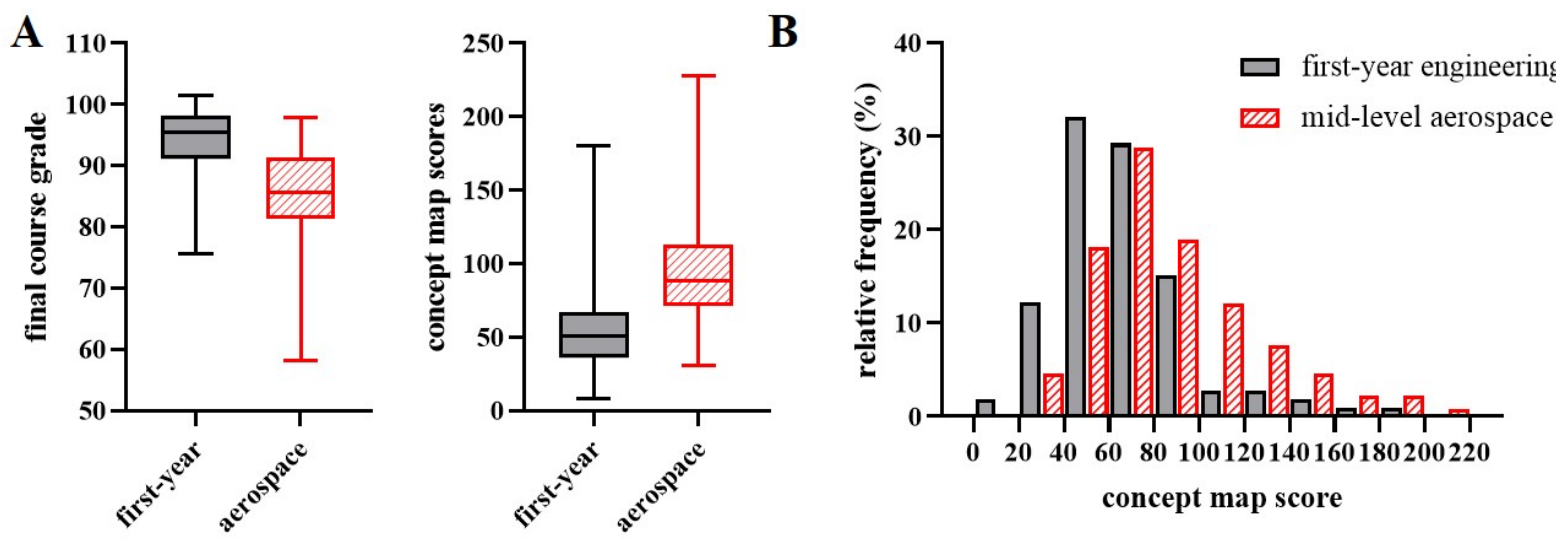

Figure 2. A. Box-and-whisker plots show horizontal lines representing the first (lower) quartile, median, and third (upper) quartile of the data; whiskers represent the minimum and maximum of the data set. B. Relative frequency distributions for each course show that concept map scores are non-normally distributed $(\mathrm{p}<0.001)$. Bins are centered by 20 scoring points.

\section{Discussion}

Students' usage of a variety of techniques (e.g. central topic vs. no central topic) and creation tools (e.g. hand-written or software tool) hindered the scoring process. To minimize the variation 


\section{first-year engineering}

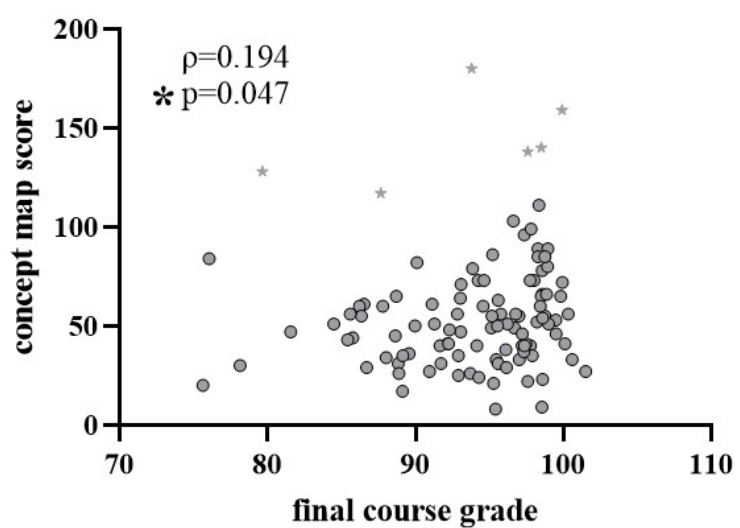

mid-level aerospace

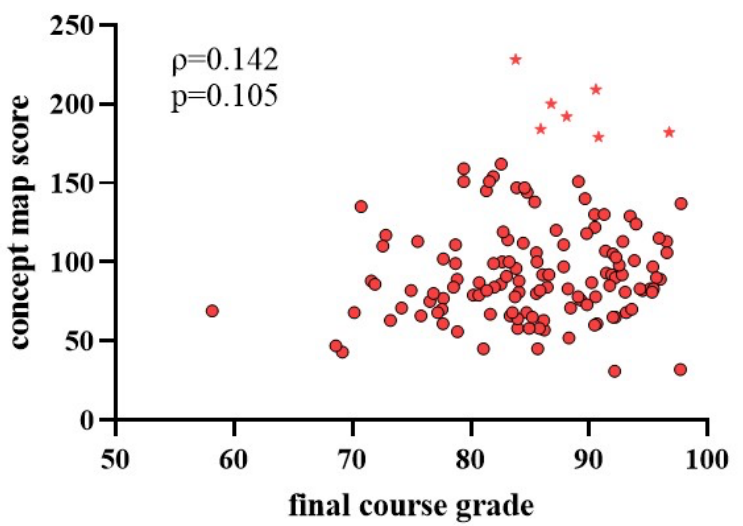

Figure 3. Scatter plots of concept map score versus final course grade show a significant yet weak correlation in the first-year engineering course and no correlation in the mid-level aerospace course. Correlation coefficients $(\rho)$ are displayed on each plot. Individual data points represent data from a single student with stars representing those identified as high magnitude outliers for concept map score.

of concept map submissions for the next iteration of this project, the assignment prompt has been modified to clarify the expectations for concept map creation. The prompt now requires students to start with the course title as the central topic and to create the concept map electronically rather than hand-written. We anticipate that these two changes will allow for easier scoring of the $\mathrm{NC}, \mathrm{HH}$, and NCL and make the determination of these components by raters less ambiguous.

We chose the traditional concept map scoring method to assess students' ability to make connections since it is an established concept map scoring method within engineering education [18] and contains a clear sub-score that quantifies the inter-hierarchy connections visible in a concept map. However, the traditional method does not contain a sub-score for intra-hierarchy connections. We observed that most of the line connections students made within a hierarchy (intra- connections) were logical and often connected course material that was introduced separately in the course timeline (e.g. "Bending" and "Moments of Inertia" in the mid-level aerospace course). Therefore, the traditional scoring method may not fully capture students' ability to make connections. As such, we are exploring the addition of a sub-score that quantifies the inter- and intra-hierarchy connections together in future iterations of our scoring method to provide a more comprehensive metric to gauge students' connection-making abilities.

Although the traditional scoring method is practical for large-scale and sustainable implementation, it is limited in its lack of accountability for the logical correctness of connected concepts (nodes) [19]. Furthermore, in their comparison of the traditional scoring method and holistic scoring method of concept maps showing student perceptions of EM, Martine, Mahoney, Sunbury, Schneider, Hixon, and Bodnar [19] suggest the traditional scoring method may be more geared toward understanding students' knowledge of a concept (versus the connectedness of concepts). They bring to light the fact that the traditional method awards points for concepts without relationship to the central topic [19]; we observed this in the present study whereby some maps contained nodes that earned points regardless of their connectedness, or lack thereof, 
to other nodes. Collectively, this prior study taken together with our present findings motivate our current efforts to adapt the traditional scoring method and/or to develop our own concept map scoring methodology to emphasize the logical correctness of map components in parallel with the NC, HH, and NCL metrics.

Overall, correlation analyses between final course grade and concept map scores (Figure 3) support that concept maps are an assessment tool that is not substantially impacted by students' course performance. This notion is supported by the lack of correlation between course grades and concept map scores in the aerospace course and a weak correlation coefficient in the firstyear engineering course $(\rho=0.194$ on a scale from $-1 \leq \rho \leq+1)$ that, although statistically significant, may not be statistically meaningful (Figure 3). Although we do not detect strong relationships between final course grades and concept map scores (Figure 3), significant relationships between concept map scoring metrics and single exam scores have been observed [20]. A prior study in an undergraduate psychology course found that the number of nodes on student-generated concept maps, when used as an optional extra-credit activity, significantly correlate with student exam scores [20]. Yet, node quantity and exam score only correlate if the exam was taken after completion of the concept map [20], suggesting that concept maps as intervention tools may themselves influence course performance. Accordingly, instructors should consider this effect if electing to use concept map prompts for assessment purposes related to EM or for other purposes, with particular attention paid to the timing of concept map prompts relative to course examinations.

The difference in the strength of correlation between the two courses in our study $(\rho=0.194$, first year; $\rho=0.142$, aerospace), as well as a 1.7 -fold increase in concept map scores for the aerospace over the first-year course (Table 1, Figure 2), raise questions about how the course content and academic year affect the complexity of connections students make. Indeed, prior work supports that course content and concept map complexity are not independent in a non-engineering course [20]. Although student majors were likely to vary more widely for the students in the first-year course (variety of engineering majors) versus in the aerospace course (primarily aerospace engineering majors), choice of engineering major is not expected to influence student concept maps scores since the assignment prompts dealt specifically with course content. Future studies will continue to investigate relationships between course grades and concept map scores, as well as explore how students' ability to make connections may change with course- and year-related variables.

\section{Conclusion}

As the integration of EM into engineering undergraduate programs grows in popularity, it is important to assess students' development of an EM to inform the efficacy of EM curriculum and course changes. While there are existing EM assessments, there has been a call for assessments that focus on the sub-constructs of EM individually (i.e. curiosity, creating value, connections) rather than holistically. Concept maps have been previously used to assess student development of an EM as a whole, but we used concept maps to specifically assess the ability of students to make connections. Collectively, we found no strong correlation between course grades and scores on concept maps developed by students for the course. This result supports the 
conclusion to move forward with the concept map scoring methodology without the need for a correction factor based on grades. However, the results of the observations taken during the scoring process indicate the need for modifications to the concept map instructions prompt and scoring method in future studies. We have found shortcomings of the traditional concept map scoring method and have discussed the holistic concept map scoring method. These shortcomings lead us to believe that neither method on its own can measure the ability to make connections well enough within the demands of use across a wide range of courses by a multitude of graders to constitute their use without revisions. For future evaluation of student ability to make connections that are conceptually meaningful, we will investigate creating a new concept map scoring method with a stronger focus on measuring connection logic. However, any new scoring method would need to be compared again to course grades to identify if the correction factor is needed. Once this new concept map scoring method is developed and validated, we can apply this scoring method in the future to measure student ability to make connections as a pre- and post-test in a single course or examine the impact of curricular changes in a course by comparing the results in different years or under different instructors.

\section{References}

1. M.T. Azim, and A.H. Al Kahtani, "Entrepreneurship Education, and Training: A Survey of Literature," Life Science Journal, vol. 11, no. 1s, 2014.

2. The Kern Family Foundation, "Engineering Unleashed," https://engineeringunleashed.com/, 2021, (accessed January 2021).

3. A. R. Peterfreund, E. Costache, H. L. Chen, S. K. Gilmartin, and S. Sheppard, "Infusing innovation and entrepreneurship into engineering education: Looking for change as seen by ASEE Members," Proceedings of the 2016 ASEE Annual Conference, New Orleans, LA, June 2016.

4. W. F. Massy, T.A. Sullivan, and C. Mackie, "Improving measurement of productivity in higher education," Change: The Magazine of Higher Learning, vol. 45, no. 1, 15-23, 2013.

5. S. R. Brunhaver, J. M. Bekki, A. R. Carberry, J. S. London, and A. F. Mckenna, "Development of the Engineering Student Entrepreneurial Mindset Assessment (ESEMA)," Advances in Engineering Education, 7(1), pp. 1-12, 2018.

6. C. Q. Li, R. S. Harichandran, M. I. Carnasciali, N. O. Erdil, and J. Nocito-Gobel, "Development of an instrument to measure the entrepreneurial mindset of engineering students," ASEE Annual Conference and Exposition, Conference Proceedings, June 2016.

7. S. E. Zappe, "Avoiding construct confusion: An attribute-focused approach to assessing entrepreneurial mindset," Advances in Engineering Education, 7(1), 1-12. 2018.

8. J. D. Novak and B. Gowin, Learning How to Learn, Cambridge University Press, New York, NY, 1984, pp. 36-37, 93-108.

9. M.K. Watson and E. Barrella, "Using concept maps to explore the impacts of a learningcycle-based sustainability module implemented in two institutional contexts," Journal of 
Professional Issues in Engineering Education and Practice, vol. 143, no.2, pp. D4016001, 2017.

10. D. Hyerle, Visual Tools for Constructing Knowledge, Association for Supervision and Curriculum Development, Alexandria, VA, 1996.

11. K. M. Edmondson, Assessing Science Understanding Chapter 2 - Assessing science understanding through concept maps, Academic Press, Pages 15-40, 2005.

12. J. Stewart, J. Van Kirk, R. Rowell, "Concept Maps: A Tool for Use in Biology Teaching," The American Biology Teacher, March 1979.

13. B.J. Daley and D.M. Torre, "Concept maps in medical education: an analytical literature review, "Medical Education, 2010.

14. T. Holme and K. Murphy, "The ACS Exams Institute Undergraduate Chemistry Anchoring Concepts Content Map I: General Chemistry," Journal of Chemical Education, 2012.

15. B.J. Daley, C.R. Shaw, T. Balistrieri, K. Glasenapp, L. Piacentine, "Concept maps: a strategy to teach and evaluate critical thinking," J Nurs Educ., January 1999.

16. C.G. Williams, "Using Concept Maps to Assess Conceptual Knowledge of Function," Journal for Research in Mathematics Education, vol. 29, no. 4, pp. 414-421, 1998.

17. C. Bodnar, S. Jadeja, and E. Barrella, "Creating a Master 'Entrepreneurial Mindset' Concept Map," Proceedings of the 2020 ASEE Annual Conference, Virtual, 2020.

18. M. K. Watson, J. Pelkey, C. R. Noyes, and M. O. Rodgers, "Assessing Conceptual Knowledge Using Three Concept Map Scoring Methods," Journal of Engineering Education, 105(1), 118-146. 2016.

19. M. M. Martine, L. X. Mahoney, C. M. Sunbury, J. A. Schneider, C. Hixson, and C. A. Bodnar, "Concept maps as an assessment tool for evaluating students' perception of entrepreneurial mind-set," ASEE Annual Conference and Exposition, Conference Proceedings. 2019.

20. J. W. Berry and S. L. Chew, "Improving Learning through Interventions of StudentGenerated Questions and Concept Maps," Teach. Psychol., vol. 35, no. 4, pp. 305-312, 2008. 